\title{
Role of Maternal Serum Ferritin in the Prediction of Asymmetric Intrauterine Growth Restriction.
}

\author{
Original \\ Article \\ Shaimaa M Salem ${ }^{1}$, Sherif A Ashoush ${ }^{2}$, Hassan A Bayoumi', Haitham A Elsabaa ${ }^{2}$ \\ ${ }^{I}$ Department of Obstetrics and Gynecology, Damietta Specialized Hospital, Damietta \\ ${ }^{2}$ Department of Obstetrics and Gynecology, Faculty of Medicine, Ain Shams University
}

\begin{abstract}
Aim: Asymmetric intrauterine growth restriction (IUGR) carries increased intrauterine and neonatal risks. Since most cases are idiopathic, identifying a predictive test remains an ideal prevention management. Maternal serum ferritin level is a potential predictor of later developing asymmetric IUGR.

Patients and Methods: Blood samples were drawn, at 30-32 gestational weeks, from 450 women who were then followedup resulting in 32 term pregnancies with asymmetric IUGR. The control group included the first recruited 32 women delivering adequate for gestational age neonates at term. Serum ferritin was then measured in the stored serum samples. Ultrasound scanning was performed at 30-32 weeks then at 37 weeks. Umbilical and middle cerebral artery Doppler scans were added at 37 weeks.

Results: Serum ferritin, at 30-32 weeks, was higher in women delivering IUGR babies (19.3 \pm 6.83 vs $14 \pm 5.18, p<0.01)$. At 37 weeks, pregnancies with asymmetric IUGR had significantly $(\mathrm{p}<0.01)$ higher umbilical artery resistance and pulsatility indices ( $0.71 \pm 0.06$ vs $0.59 \pm 0.07$ and $1.24 \pm 0.17$ vs $0.86 \pm 0.09$, respectively). Their middle cerebral artery RI and PI were significantly $(\mathrm{p}<0.01)$ lower than controls $(0.74 \pm 0.09$ vs $0.81 \pm 0.05$ and $1.64 \pm 0.07$ vs $1.9 \pm 0.15$, respectively). Ferritin level correlated significantly with neonatal birthweight. A cutoff level $>18.2 \mathrm{ng} / \mathrm{mL}$ had a sensitivity of $59.4 \%$ and a specificity of $90.6 \%$. The area under curve showed an accuracy of $76.8 \%$. Women with ferritin $>18.2 \mathrm{ng} / \mathrm{ml} \mathrm{were} 10.23$ times more likely to get asymmetrically growth restricted neonates (CI 2.89-36.17, $\mathrm{p}<0.001)$.

Conclusion: This study presents further evidence that maternal serum ferritin may be a useful test in the prediction of asymmetric IUGR.
\end{abstract}

Key Words: Intrauterine growth restriction, doppler, ferritin, neonatal birthweight, small for gestational age.

Received: 14 May 2019, Accepted: 7 June 2019

Corresponding Author: Sherif A Ashoush, MD, Department of Obstetrics and Gynecology, Faculty of Medicine, Ain Shams University, Cairo, Egypt, Tel.: +201222660266, E-mail: sherifashoush@med.asu.edu.eg

ISSN: 2090-7265, August 2019, Vol. 9, No. 3

\section{INTRODUCTION}

Asymmetric, late-onset (type II), intrauterine growth restriction (IUGR) may be defined as pathological slow fetal growth, beginning in late pregnancy, due to uteroplacental insufficiency ${ }^{[1,2]}$. This definition differentiates it from the term, Small for Gestational Age (SGA), which implies a birthweight less than the $10^{\text {th }}$ centile for gestational age even if it is a healthy but simply small baby. 2 The incidence of IUGR is $3.3-10 \%$ in the developed countries and $6.7-17 \%$ in developing ones ${ }^{[3,4,5]}$.

A fetus with IUGR is exposed to increased intrauterine risks of fetal distress and death, neurologic developmental disorders as well as meconium aspiration at birth. Neonatal risks include hypoglycemia, long admission to intensive care units, hypothermia, polycythemia, jaundice, feeding difficulties, necrotizing enterocolitis, late-onset sepsis, hypoxic-ischemic encephalopathy and pulmonary hemorrhage. These infants also have increased risks of type 2 diabetes, obesity, autoimmune diseases, cardiovascular diseases and hypertension in adult life ${ }^{[2,6]}$.

Asymmetric IUGR, accounting for $70-80 \%$ of cases of IUGR, ${ }^{[2]}$ is associated with reduced umbilical blood flow, brain sparing effect, oligohydramnios and a low Ponderal index. ${ }^{[7]}$ In the absence of any direct causal therapy and in view that most cases of IUGR are idiopathic, the identification of adequate predictive tests for IUGR remains one of the top priorities in obstetrics. Several studies suggested different biomolecules as early markers for IUGR, such as lactate dehydrogenase, leptin, metastin, adiponectin, s-endoglin, endothelin-1 and pregnancy associated plasma protein. ${ }^{[8-10]}$ Most of these tests have a low sensitivity or are expensive, not widely available and/or invasive tests requiring amniocentesis. ${ }^{[9-10]}$ 
Ferritin, the main iron storage protein, was suggested to be an adequate alternative as a screening test being a relatively cheap and easily available blood test. Its level is known to rise in response to hypoxia or as an acute phase reactant in infections. ${ }^{[1]}$ Ferritin blood level normally drops, in correlation with the progressive depletion of iron reserves, by $32 \%$ during the first trimester, $39 \%$ in the second and up to $53 \%$ during the third trimester. ${ }^{[12]}$ This level reaches a nadir at 30-32 weeks, ${ }^{[13]}$ after which it stays constant.

The theory behind the rise in maternal serum level of ferritin during pregnancies destined to develop lateonset (asymmetric). IUGR relies on decreased placental extraction of ferritin from the systemic circulation. ${ }^{[13]}$ Its predictive value was previously investigated, in a few small-sized studies, on a small number of cases. ${ }^{[9,13-16]}$

This study aimed to assess the accuracy of maternal serum ferritin level at 30 weeks to 32 weeks in predicting the development of IUGR and to identify the ferritin cutoff level with the best predictive value, in a well-selected, adequate-sized group of pregnant women.

\section{PATIENTS AND METHODS}

This was a prospective nested longitudinal study conducted in the outpatient clinics of Ain-Shams University Maternity Hospital, Cairo, Egypt. The study protocol was approved by the Faculty of Medicine Research Review Board on 15/03/2016. The study protocol was registered on 14/04/2016 on www.clinicaltrials.com website as NCT02738463. All women were informed about the procedures of the study. Confidentiality of the collected data and anonymity of the participants were ensured. Written informed consent was obtained from the women before participation. The study protocol complied with the Declaration of Helsinki regarding ethical conduct of research involving human subjects.

All included women had singleton 30-32 weeks' pregnancies (with sure and reliable dates of the last menstrual periods and ultrasonographic estimation of the gestational age during the first trimester). Exclusion criteria included history of anemia, iron supplementation, recent blood transfusion, age $<20$ years, BMI $<18$, smoking, diabetes mellitus during pregnancy, hepatic, renal, hypertensive or cardiovascular abnormalities, detected congenital fetal anomalies, antepartum hemorrhage, preterm delivery and cases developing acute infections during pregnancy with raised leukocytic count or C-reactive protein. This was meant to rule out any confounding effect on the level of serum ferritin.

\section{Sample size calculation:}

Data from a previous study showed that maternal serum ferritin levels in pregnancies resulting in neonates with
IUGR were on average $6.43 \mathrm{mg} / \mathrm{L}$ higher than in those with adequate sized neonates. ${ }^{[15]}$

Using the equation of sample size calculation from Fox et $a l,{ }^{[17]}$ with a power of $80 \%(\beta=0.2)$ and a confidence level of $95 \%(\alpha=0.05)$, the sample size calculation for the study group is as follows:

$$
\begin{aligned}
& \mathrm{N}=\mathrm{D} \times \mathrm{K} \times 2 \times[\partial /(\mathrm{f} 1-\mathrm{f} 2)]^{2} \\
& =133.3 \% \times 7.9 \times 2 \times\left[7.84 /(17.54-11.11]^{2}\right. \\
& =32 \text { cases per group }
\end{aligned}
$$

Where $\mathrm{N}=$ Sample size (in a single arm), $\mathrm{K}=$ Constant, which varies according to $\alpha$ and $\beta$ values, here $K=7.9$, $\partial=$ standard deviation in ferritin level, and $\mathrm{f}_{1}$ and $\mathrm{f}_{2}=$ the ferritin level among cases versus controls in a previously published study (15). $\mathrm{D}=$ percentage increase to compensate for the drop-out rate, here $\mathrm{D}=133.3 \%$.

Assuming an incidence of IUGR of $8 \%,{ }^{[15]} 400$ patients were to be recruited for follow up to obtain the desired 32 cases with IUGR. Another $10 \%$ rate was added to that number of women to ascertain obtaining the desired number of IUGR cases during patient follow-up. Thus, a total of 450 patients were assessed and followed-up. The study group included patients delivering at $\geq 37$ weeks, giving birth to neonates with type II IUGR (32 pregnancies). The control group included the first recruited 32 women delivering adequate for gestational age neonates at term.

Morning fasting blood samples were drawn from the cubital vein at 30-32 weeks. The specimens were centrifuged immediately for 5 minutes at $4000 \mathrm{rpm}$ and the supernatant serum was transferred into another Eppendorf tube and immediately frozen and stored at -20C in the biochemistry laboratory of Ain-Shams University Hospitals.

Obstetric ultrasound scanning was performed on all included women at 30-32 weeks and was repeated at 37 weeks. Umbilical and middle cerebral artery Doppler scans were added at 37 weeks in IUGR cases (the first 32 women diagnosed with IUGR) as well as controls (the first 32 normal pregnancies who reached maturity).

Pregnant women were followed until delivery to identify those with asymmetric IUGR. Serum ferritin levels were then measured in their stored samples of cases and controls using enzyme-linked fluorescent assay. The result of their complete blood count at delivery was also recorded.

Neonates were then evaluated at birth for vitality using the Apgar score (0-10) which checks the heart and respiratory rates, the muscle tone, reactivity and skin color. Neonatal weight and body length were also measured and documented, as well as any clinical evidence of IUGR, 
such as raised head to abdomen circumferences ratio, loose and dry skin, absent buccal fat (old man look), small or scaphoid abdomen, thin umbilical cord often stained with meconium, excessive skin folds with decreased underlying skeletal muscle mass and loss of subcutaneous fat.

Data were collected, revised, coded and entered to the Statistical Package for Social Science program (IBM Corp. Released 2016. IBM SPSS Statistics for Windows, Version 24. Armonk, NY: IBM Corp). Qualitative data were presented as number and percentage and were compared using Chi-square $x 2$ test or Fisher exact test. Quantitative parametric data were presented as mean \pm standard deviation $( \pm \mathrm{SD})$ and were compared using Independent t-test. Non-parametric data were presented as median, with interquartile ranges $(I Q R)$, and were compared using Mann-Whitney test. Spearman correlation coefficients were used to assess the correlation between two quantitative parameters in the same group. Logistic regression analysis was used to assess predictors of IUGR. Receiver operating characteristic curve (ROC) was used to assess the best cutoff point for ferritin in predicting IUGR with its area under curve (AUC), sensitivity, specificity, positive predictive value (PPV) and negative predictive value (NPV).

\section{RESULTS}

A total of 450 women were recruited during the course of this study. Four pregnancies ended with intrauterine death, ten women developed gestational diabetes mellitus, 28 developed preeclampsia, 32 delivered prematurely and 48 were lost to follow up. Out of the remaining 328 pregnancies, the first 32 cases of IUGR and 32 adequate for gestational age (AGA) controls were included in data analysis. The mean age of the included women were statistically similar $(28 \pm 4.1$ and $28.4 \pm 4$, in controls and cases, respectively, $\mathrm{p}=0.7$ ). Both groups had comparable rates of previous IUGR deliveries (6.3 and $17 \%$ respectively, $\mathrm{p}=0.17$ ).

Women with asymmetric IUGR had similar hemoglobin level, hematocrit value erythrocytic and leukocytic counts as controls (Table 1). Serum ferritin at 30-32 weeks was significantly higher in women who ended up delivering IUGR babies (Table 1). At the 37 weeks' Doppler scan, pregnancies with asymmetric IUGR had significantly higher umbilical artery resistance indices (RI) and pulsatility indices (PI). Their middle cerebral artery RI and PI were significantly lower than their controls (Table 1).

Table 1: Comparison between cases and controls regarding laboratory and Doppler variables.

\begin{tabular}{|c|c|c|c|c|c|}
\hline & & & $\begin{array}{c}\text { Cases group } \\
n=32\end{array}$ & $\begin{array}{c}\text { Control group } \\
n=32\end{array}$ & Pvalue \\
\hline \multirow{2}{*}{\multicolumn{2}{|c|}{ Erythrocytes $\left(10^{6} / \mu 1\right)$}} & Mean $( \pm \mathrm{SD})$ & $4.12( \pm 0.58)$ & $3.88( \pm 0.66)$ & 0.13 \\
\hline & & Range & $3.2-5.23$ & $3.04-5.04$ & \\
\hline \multirow{2}{*}{\multicolumn{2}{|c|}{ Hemoglobin (gm/dl) }} & Mean $( \pm \mathrm{SD})$ & $11.5( \pm 0.43)$ & $11.4( \pm 0.36)$ & 0.12 \\
\hline & & Range & $11-12.5$ & $11-12.3$ & \\
\hline \multirow{2}{*}{\multicolumn{2}{|c|}{ Hematocrit (\%) }} & Mean $( \pm \mathrm{SD})$ & $34.6( \pm 1.84)$ & $33.9( \pm 1.59)$ & 0.12 \\
\hline & & Range & $31.3-38.6$ & $30.8-37.8$ & \\
\hline \multirow{2}{*}{\multicolumn{2}{|c|}{ Leucocytes $\left(10^{3} / \mathrm{cc}\right)$}} & Mean $( \pm \mathrm{SD})$ & $8.6( \pm 2.13)$ & $8.5( \pm 1.76)$ & 0.84 \\
\hline & & Range & $5.4-12.3$ & $5.4-12.2$ & \\
\hline \multirow[t]{2}{*}{ Ferritin (ng/ml) } & & Mean $( \pm \mathrm{SD})$ & $19.3( \pm 6.83)$ & $14( \pm 5.18)$ & $<0.01$ \\
\hline & & Range & $8.5-40$ & $5-26.3$ & \\
\hline Umbilical & RI & Mean $( \pm \mathrm{SD})$ & $0.71( \pm 0.06)$ & $0.59( \pm 0.07)$ & $<0.01$ \\
\hline artery & & Range & $0.6-0.81$ & $0.49-0.69$ & \\
\hline \multirow[t]{2}{*}{ Doppler } & PI & Mean $( \pm \mathrm{SD})$ & $1.24( \pm 0.17)$ & $0.86( \pm 0.09)$ & $<0.01$ \\
\hline & & Range & $1.03-1.94$ & $0.72-0.94$ & \\
\hline Middle & RI & Mean $( \pm \mathrm{SD})$ & $0.74( \pm 0.09)$ & $0.81( \pm 0.05)$ & $<0.01$ \\
\hline cerebral & & Range & $0.61-0.92$ & $0.72-0.89$ & \\
\hline artery & PI & Mean $( \pm \mathrm{SD})$ & $1.64( \pm 0.07)$ & $1.9( \pm 0.15)$ & $<0.01$ \\
\hline Doppler & & Range & $1.36-1.88$ & $1.82-2.11$ & \\
\hline
\end{tabular}

$\mathrm{N}=$ number of women, $\mathrm{SD}=$ standard deviation, $\mathrm{RI}=$ resistance index, $\mathrm{PI}=$ pulsitility index.

Neonates with asymmetric IUGR had a similar mode of delivery and gestational age at birth as their AGA controls. On the other hand, the birthweight, Apgar scores and rate of admission to neonatal intensive care units were all significantly different between IUGR and AGA neonates (Table 2). 
Table 2: Comparison between cases and controls regarding neonatal outcome at delivery.

\begin{tabular}{llccc}
\hline & & Cases group & Control group & P value \\
& & $\mathrm{n}=32$ & $\mathrm{n}=32$ & 0.14 \\
Gestational age at delivery (days) & Mean $( \pm \mathrm{SD})$ & $272.9( \pm 7.25)$ & $274.3( \pm 6.79)$ & $260-285$ \\
& Range & $259-282$ & $16,50 \%$ & 0.45 \\
Mode of delivery (n, \%) & Cesarean & $19,59.4 \%$ & $16,50 \%$ & $<0.001$ \\
& Vaginal & $13,40.6 \%$ & $3419( \pm 352)$ & 0.04 \\
Birth Weight $(\mathrm{gm})$ & Mean $( \pm \mathrm{SD})$ & $2134( \pm 143)$ & $2800-4100$ & \\
& Range & $1750-2390$ & $8(7-9)$ & 0.03 \\
Apgar score $\left(1^{\text {st }}\right.$ minute) & Median $(I Q R)$ & $7(7-8)$ & $6-10$ & \\
Apgar score $\left(5^{\text {th }}\right.$ minute) & Range & $6-9$ & $9(9-10)$ & $8-10$ \\
Admission to NICU (n, \%) & Median $(I Q R)$ & $8.5(8-9.5)$ & $28,87.5 \%$ & 0.01 \\
& Range & $7-10$ & $4,12.5 \%$ & \\
\hline
\end{tabular}

$\mathrm{N}=$ number of women, $\mathrm{SD}=$ standard deviation, $\mathrm{IQR}=$ interquartile range, $\mathrm{NICU}=$ neonatal intensive care unit.

Maternal serum ferritin level was the only variable which correlated significantly with neonatal birthweight. Maternal age and hematology variables, neonatal gestational age,
Apgar scores or body length were all not correlated to ferritin level (Table 3).

Table 3: Correlation between serum ferritin level and maternal and neonatal data in cases and controls.

\begin{tabular}{|c|c|c|c|c|}
\hline & \multicolumn{4}{|c|}{ Ferritin level (ng/ml) } \\
\hline & \multicolumn{2}{|c|}{ Cases } & \multicolumn{2}{|c|}{ Controls } \\
\hline & rvalue & P value & rvalue & Pvalue \\
\hline Maternal Age (years) & -0.195 & 0.28 & -0.112 & 0.54 \\
\hline Erythrocytes $(106 / \mu 1)$ & 0.111 & 0.55 & -0.073 & 0.69 \\
\hline Hemoglobin (gm/dl) & 0.080 & 0.66 & 0.148 & 0.42 \\
\hline Hematocrit (\%) & 0.225 & 0.22 & 0.044 & 0.81 \\
\hline Leucocytes $\left(10^{3} / \mathrm{cc}\right)$ & 0.250 & 0.17 & -0.109 & 0.56 \\
\hline Gestational age at delivery (days) & -0.261 & 0.15 & 0.103 & 0.57 \\
\hline Birth Weight (gm) & -0.453 & 0.009 & 0.174 & 0.34 \\
\hline Apgar score (1st minute) & -0.268 & 0.14 & 0.063 & 0.73 \\
\hline Apgar score (5th minute) & -0.090 & 0.63 & 0.083 & 0.65 \\
\hline
\end{tabular}

$r$ value $=$ Spearman coefficient of correlation

There was a non-significant correlation between the history of IUGR in previous pregnancies and the development of asymmetric IUGR in the current pregnancy. A highly significant correlation was noted between serum ferritin level at 30-32 weeks as well as Doppler indices at 37 weeks and present history of asymmetric IUGR (Table 4).

Table 4: Correlation between the development of IUGR in the current pregnancy and the past history of previous IUGR deliveries, current pregnancy serum ferritin level or umbilical and middle cerebral artery Doppler indices.

\begin{tabular}{lcc}
\hline & Development of IUGR in the current pregnancy & P value \\
\hline Previous IUGR & $r$ value & 0.09 \\
Ferritin (ng/ml) & 1.435 & 0.001 \\
Umbilical artery RI & 0.183 & $<0.001$ \\
Umbilical artery PI & 16.866 & $<0.001$ \\
Middle cerebral artery RI & 6.596 & 0.002 \\
Middle cerebral artery PI & -12.308 & 0.03 \\
\hline
\end{tabular}

$r$ value $=$ Spearman coefficient of correlation.

$\mathrm{RI}=$ resistance index, $\mathrm{PI}=$ pulsitility index. 
The receiver operating characteristic (ROC) curve (Table 5, Fig. 1) showed that the best ferritin cutoff level, between mothers with asymmetric IUGR neonates and those with AGA, was $>18.2 \mathrm{ng} / \mathrm{mL}$. This cutoff had a sensitivity of $59.38 \%$ and a specificity of $90.62 \%$. The area under curve (AUC) showed an accuracy of $76.8 \%$, thus serum ferritin was found a good predictor for predicting babies with IUGR. The data above depict that women with mean serum ferritin above $18.2 \mathrm{ng} / \mathrm{ml}$, were 10.23 times more likely to get asymmetrically growth restricted babies (CI 2.89-36.17, $\mathrm{p}<0.001$ ).

Table 5: Data showing sensitivity, specificity, positive predictive value, negative predictive value of various serum cutoffs to predict asymmetrical IUGR.

\begin{tabular}{|c|c|c|c|c|c|}
\hline Cutoff point & AUC & Sensitivity & Specificity & $P P V$ & $N P V$ \\
\hline$>14.7$ & 0.734 & 75.00 & 71.87 & 72.7 & 74.2 \\
\hline$>15.6$ & 0.687 & 65.62 & 71.87 & 70.0 & 67.6 \\
\hline$>16.4$ & 0.719 & 65.62 & 78.12 & 75.0 & 69.4 \\
\hline$>17$ & 0.703 & 59.38 & 81.25 & 76.0 & 66.7 \\
\hline$>18.2$ & 0.768 & 59.38 & 90.62 & 86.4 & 69.0 \\
\hline$>20.1$ & 0.625 & 34.38 & 90.62 & 78.6 & 58.0 \\
\hline$>22$ & 0.641 & 34.38 & 93.75 & 84.6 & 58.8 \\
\hline$>25$ & 0.516 & 9.38 & 93.75 & 60.0 & 50.8 \\
\hline$>26$ & 0.531 & 9.38 & 96.87 & 75.0 & 51.7 \\
\hline
\end{tabular}

$\mathrm{AUC}=$ area under the curve, $\mathrm{PPV}=$ positive predictive value, $\mathrm{NPV}=$ negative predictive value.

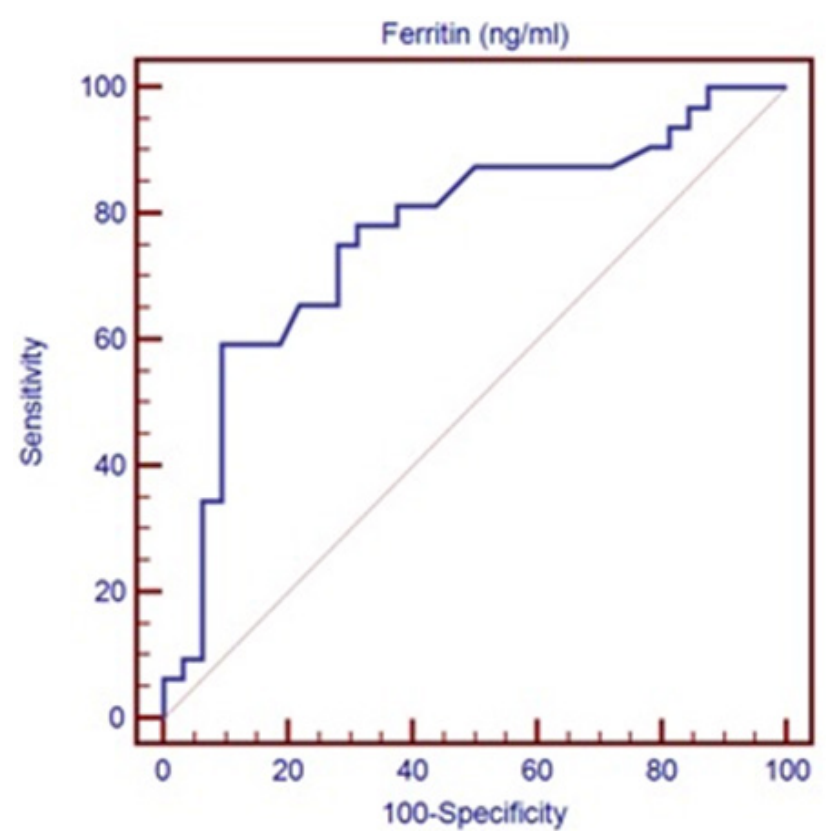

Fig. 1: Receiver operating characteristic curve showing the best cutoff point for serum ferritin level between mothers with asymmetric IUGR neonates and those with AGA, was $>18.2$ $\mathrm{ng} / \mathrm{mL}$.

\section{DISCUSSION}

Fetal growth restriction is a serious condition deserving early diagnosis or good predictive tests to help reduce the impact of its serious short and long term complications. The current study found a significantly higher mean maternal serum ferritin level in the group of pregnant women who ended up being diagnosed with IUGR, as opposed to that in control pregnancies $(19.3 \pm 6.83$ vs. $14 \pm 5.18 \mathrm{mg} / \mathrm{L}$, respectively; $\mathrm{p}<0.001)$. This is, roughly, similar to the so far recorded ferritin levels in similar studies. The value of maternal serum ferritin in predicting asymmetric IUGR was previously investigated, for few times, on small numbers of cases, i.e. 7 cases in Ozgu-Erdinc et al. ${ }^{[9]}$, 18 cases in Milasinovic et al $.^{[13]}, 10$ cases in Hubel et al. ${ }^{[14]}, 17$ cases in Visnjevac et al. ${ }^{[15]}$, and 31 cases in Bindal et al., ${ }^{[16]}$ or with limitations in the research methodology such as measuring ferritin at a gestational age that was to early (20 weeks) or to late (36 weeks) ${ }^{[1,4]}$, or including cases with maternal iron deficiency anemia ${ }^{[15]}$.

Lower expression of placental transferrin is known to be associated with IUGR ${ }^{[18]}$. This may cause less iron extraction by the placenta from maternal serum, thus raising the level of serum ferritin ${ }^{[16]}$. Furthermore, other studies suggest that placental damage, in cases of asymmetric IUGR, releases some of the placental ferritin into the maternal circulation, thus further raising its serum level ${ }^{[1,14,19]}$. Ferritin is also a known acute phase reactant released by leukocytes into the systemic circulation in response to immune activation. High serum ferritin level may reflect a non-infectious vascular inflammatory response causing oxidative damage to cells and tissues ${ }^{[14,20]}$. The association of IUGR with inflammation has been previously proven. ${ }^{[21]}$ This is the reason why this study set acute infections during pregnancy, leukocytosis or raised C-reactive protein titer among its exclusion criteria to rule out any misleading rise in the level of ferritin.

As opposed to previous studies ${ }^{[3,15,16]}$, we set anemia among our exclusion criteria. Our results showed non-significant differences between controls and IUGR cases as regards erythrocytic counts, hematocrit and hemoglobin levels, compared to a rate of anemia of $47.4 \%$ in the control group of one study ${ }^{[15]}$. This is probably why the current study 
found a higher degree of correlation between maternal serum ferritin and neonatal birthweight $(-0.453)$ which is higher than those found by Višnjevac et al. $(-0.24),{ }^{[15]}$ and Bindal et al. (-0.36). ${ }^{[16]}$ On other grounds, Akkurt et al. reported a higher hematocrit level in pregnancies with IUGR than controls. ${ }^{[1]}$ They hypothesized that this was related to a degree of hemoconcentration related to IUGR development. The current study did not show such difference in hematocrit.

We showed maternal serum ferritin to have a good predictive power when used at 30-32 weeks for prediction of IUGR. The results of the current study suggest that a ferritin level of $>18.2 \mathrm{ng} / \mathrm{mL}$ can predict asymmetric IUGR with a sensitivity of $59.38 \%$, a specificity of $90.62 \%$, a positive predictive value of $86.4 \%$ and a negative predictive value of $69 \%$. The very good overall accuracy of $76.8 \%$ drawn from the ROC analysis and the associated odds ratio of 10.23 are promising, denoting that serum ferritin measurement may be considered an adequate predictive test for IUGR. Uberos et al., Višnjevac et al. and Bindal et al. also came to similar conclusions but with a lower odds ratio, ${ }^{[3,15,16]}$ probably due to the inclusion of cases with anemia in the study population. ${ }^{[3,16]}$ Uberos et al. concluded that ferritin levels $>13 \mathrm{ng} / \mathrm{ml}$ had 4.5 times more risk to result in IUGR at 38 weeks of gestation. ${ }^{[3]}$ Hubel et al. showed no significant difference in ferritin between AGA and IUGR cases, but their study only included 10 asymmetric IUGR cases. ${ }^{[14]}$ Soubasi et al. found high maternal ferritin levels to correlate significantly with higher rates of gestational diabetes mellitus and IUGR, but did not define a cutoff to differentiate IUGR from AGA neonates. ${ }^{[22]}$ Hou et al., Milasinovic et al. and Višnjevac et al. identified the ferritin threshold to predict IUGR or SGA as $>13.6 \mathrm{ng} / \mathrm{ml} \cdot .^{[4,13,15]}$ Ozgu et al. reported maternal serum ferritin levels $>60 \mathrm{ng} / \mathrm{ml}$ to increase the risk for IUGR. ${ }^{\left[{ }^{[]}\right.}$Bindal et al. concluded that maternal serum ferritin $>20 \mathrm{ng} / \mathrm{ml}$ predicted IUGR with an odds ratio of 6.6. ${ }^{[16]}$ Akkurt et al. took one step further and showed a role for maternal serum ferritin measurements in distinguishing pregnancies with asymmetric IUGR from those with simple small for gestational age babies and no signs of placental insufficiency. ${ }^{[1]}$

In conclusion, this study presents further evidence on a relatively larger group of patients, that maternal serum ferritin might serve as a useful marker to identify pregnancies at risk of resulting in asymmetric IUGR infants. It found a significantly higher maternal serum ferritin level, at 3032 weeks, in pregnancies destined to develop asymmetric IUGR at a later gestational age than in controls. A cutoff of $>18.2 \mathrm{ng} / \mathrm{mL}$ had an accuracy of $76.8 \%$ to predict IUGR with a PPV of $86.4 \%$. Pregnancies with ferritin level higher than such cutoff were 10.23 times more likely to deliver asymmetrically growth restricted babies when compared to women with serum ferritin value less then $<18.2 \mathrm{ng} / \mathrm{ml}$. Large scale studies are recommended to further establish this theory and to test related points regarding the effect of associated anemia or the gestational age at which the test is best to be done. The role of maternal serum ferritin in distinguishing pregnancies with asymmetric IUGR and signs of placental insufficiency from those with simple small for gestational age babies also still needs further research.

\section{FUNDING STATEMENT:}

This study was self-funded by the authors.

\section{CONFLICTS OF INTEREST}

There are no conflicts of interest.

\section{REFERENCES}

1. Akkurt MO, Akkurt I, Altay M, et al. Maternal serum ferritin as a clinical tool at 34-36 weeks' gestation for distinguishing subgroups of fetal growth restriction. J Matern Fetal Neonatal Med. 2017; 30(4): 452-456.

2. Sharma D, Shastri S, Sharma P. Intrauterine Growth Restriction: Antenatal and Postnatal Aspects. Clin Med Insights Pediatr. 2016; 10: 67-83.

3. Uberos J, Molina A, Munoz A. Blood ferritin levels in pregnant women as an estimator of low birth weight? Prenat Neonat Med 2000; 5: 177-181.

4. Hou J, Cliver S, Tamura T, et al. Maternal serum ferritin and fetal growth. Obstet Gynecol 2000; 95: 447-452.

5. Christian P, Khatry SK, Katz J, et al. Effects of alternative maternal micronutrient supplements on low birth weight in rural Nepal: double blind randomised community trial. BMJ 2003; 326: 571-578.

6. Jansson T, Powell TL. Role of the placenta in fetal programming: underlying mechanisms and potential interventional approaches. Clin Sci 2007; 113 (1): $1-13$.

7. Sharma D, Farahbakhsh $\mathrm{N}$, Shastri $\mathrm{S}$, et al. Intrauterine growth restriction-part 2. J Matern Fetal Neonatal Med. 2016; 29(24): 4037-4038.

8. Albu AR, Anca AF, Horhoianu VV, et al. Predictive factors for intrauterine growth restriction. J Med Life. $2014 ;$ 7(2): 165-171.

9. Ozgu-Erdinc AS, Cavkaytar S, Aktulay A, et al. Mid-trimester maternal serum and amniotic fluid biomarkers for the prediction of preterm delivery and intrauterine growth retardation. J Obstet Gynaecol Res 2014; 40(6): 1540-1546. 
10. Krantz D, Hallahan T, Janik D, et al. Maternal serum screening markers and adverse outcome: a new perspective. J Clin Med 2014; 3: 693-712.

11. Ong D, Wang L, Zhu Y, et al. The response of ferritin to LPS and acute phase of Pseudomonas infection. J Endotoxin Res. 2005; 11(5): 267-280.

12. Khambalia AZ, Collins CE, Roberts CL, et al. Iron deficiency in early pregnancy using serum ferritin and soluble transferrin receptor concentrations are associated with pregnancy and birth outcomes. Eur J Clin Nutr. 2016; 70(3): 358-363.

13. Milasinović L, Visnjevac N, Bogavac M, et al. Significance of serum ferritin level in the prediction of delivery of low birth weight newborns for gestational age. Srp Arh Celok Lek. 2013; 141(5-6): $337-343$

14. Hubel CA, Bodnar LM, Many A, et al. Nonglycosylated ferritin predominates in the circulation of women with preeclampsia but not intrauterine growth restriction. Clin Chem 2004; 50: 948-951.

15. Višnjevac N, Segedi LM, Ćurčić A, et al. Blood ferritin levels in pregnant women and prediction of the development of fetal intrauterine growth restriction. J Med Biochem. 2011; 30(4): 317-322.

16. Bindal N, Godha Z, Kohli R, et al. Role of maternal serum ferritin as a predictive marker in intrauterine growth restriction. Int $\mathrm{J}$ Reprod Contracept Obstet Gynecol. 2015; 4(3): 804-808.

17. Fox N, Hunn A, Mathers N. Sampling and sample size calculation. The National institute for health research, the NIHR Research Design Service for the East Midlands / Yorkshire \& the Humber, 2007. Available online at: www.rds-yh.nihr.ac.uk/ wp-content/uploads/2013/05/12_Surveys_and Questionnaires Revision 2009.pdf (Accessed on June 10, 2018).

18. Mandò C, Tabano S, Colapietro $\mathrm{P}$, et al. Transferrin receptor gene and protein expression and localization in human IUGR and normal term placentas. Placenta. 2011;32(1): 44-50.

19. Bothwell TH. Iron requirements in pregnancy and strategies to meet them. Am J Clin Nutr 2000; 71 (1): 257-264.

20. Scanlon KS, Yip R, Schieve LA, et al. High and low hemoglobin levels during pregnancy: differential risks for preterm birth and small for gestational age. Obstet Gynecol 2000; 96: 741-748.

21. Rao R, Georgieff M. Iron in fetal and neonatal nutrition. Sem Fetal Neonatal Med 2007; 12: 54-63.

22. Soubasi V, Petridou S, Sarafidis K. Association of increased maternal ferritin levels with gestational diabetes and intra-uterine growth retardation. Diabetes and Metabolism, 2010; 36: 58-63. 